\title{
Proposal of a Modified Comprehensive Technique to Avoid Local Scarring and Adhesion Formation for Isolated Gastrocnemius Recession: a Comparison Study.
}

Jiafa Zheng

Second People's Hospital of Dalian https://orcid.org/0000-0002-1625-5927

Xiufeng Song

The Second People's Hospital of Dalian

Min Guan

Dalian Second People's Hospital

Zhiming Qi ( 1438190369@qq.com )

Dalian Second People's Hospital

\section{Research}

Keywords: gastrocnemius contracture, gastrocnemius recession, scar adhesion, complications, control study

Posted Date: July 14th, 2021

DOI: https://doi.org/10.21203/rs.3.rs-700251/v1

License: (9) This work is licensed under a Creative Commons Attribution 4.0 International License.

Read Full License 


\section{Abstract}

\section{Background}

Poor cosmesis is one of the complications following surgical gastrocnemius recession by Strayer procedure. Our present study reported a modified comprehensive technique avoiding skin adhesion in releasing gastrocnemius contracture. A comparison cohort was conducted with inclusion cases via comprehensive modified technique and conventional Strayer procedure.

\section{Methods}

From July 2017 to December 2019, 72 consecutive patients (84 feet) were retrospectively reviewed with 38 cases $₫ 42$ feet $₫$ treated utilizing conventional Strayer procedure and 34 cases $₫ 42$ feet $\$ treated by modified comprehensive technique. All patients were followed up for minimum 12 months (mean 13.6, range 12 to 18 months). The ankle dorsiflexion range of motion and medical complications (scar adhesion) of above patients were recorded and evaluated.

\section{Results}

The mean ankle dorsiflexion significantly improved from $14.5^{\circ} \pm 2.5^{\circ}$ degrees preoperatively to $-18.7^{\circ} \pm 3.2^{\circ}$ degrees in modified procedure group postoperatively $(P<0.05)$, while dorsiflexion improved from $15.2^{\circ} \pm 3.0^{\circ}$ to $-19.1^{\circ} \pm 3.9^{\circ}$ in conventional Strayer procedure group. 1 of $42(2.4 \%)$ in modified procedure group developed scar adhesion while 12 of $42(28.6 \%)$ in conventional Strayer procedure group, which showed a statistically significant difference in postoperative complication (scar adhesion) rate between two groups $(P<0.05)$.

\section{Conclusions}

Gastrocnemius recession with modified comprehensive technique can completely release the gastrocnemius aponeurosis and achieve satisfactory recovery of ankle dorsiflexion angle, especially effectively avoid postoperative local scar adhesion with a superior cosmetic appearance.

\section{Level of Clinical Evidence: 3}

\section{Background}

The gastrocnemius contracture is often encountered in patients with foot and ankle pathology, and a higher incidence was identified especially for noninvasive etiology including hallux valgus, flat foot and so on (1-6). Three procedures of surgical gastrocnemius recession are commonly performed, including Vulpius procedure, Strayer procedure and Baumann procedure (7-16). Strayer procedure, which releases proximal medial gastrocnemius, is most popular method in the past few years, whereas postoperative complications such as poor cosmesis are often unavoidable, with a rather high complication rates of sural nerve injury, hematoma formation, strength deficits and contracture recurrence (17-26). 
Due to the tethering of the wound skin and underlying fascia, postoperative problematic scarring and adhesion formation with hypertrophic or keloid scar could cause cosmetically unsatisfactory calf and evidently affect the surgical satisfaction of gastrocnemius recession, especially among young females (16).

To the best of our knowledge, no literatures has been reported to address the cosmetic and satisfactory concern. Our present study initially proposed a novel modified comprehensive technique, by utilizing preoperative color Doppler ultrasound to accurately mark the modified incision, operating gastrocnemius recession during plantarflexion and then dorsiflexion as to address the cosmetic complications of Strayer procedure. The outcomes of current comprehensive technique were measured and analyzed, and compared with that of a conventional Strayer procedure group.

\section{Methods}

From July 2017 to December 2019, 72 patients (84 feet) from our medical center were consecutively included in this retrospective cohort study, including 4 males ( 4 feet) and 68 females ( 80 feet), of which 56 patients ( $68 \mathrm{feet}$ ) were managed with gastrocnemius recession procedure for hallux valgus, 13 patients ( 13 feet) for flatfoot and 3 patients ( 3 feet) for metatarsophalangeal joint replacement. And 38 cases(42feet)were managed with conventional Strayer procedure while 34 cases $₫ 42$ feet $\square$ were treated with modified comprehensive technique (Table 1). The most commonly used criterion to indicate an isolated gastrocnemius contracture is that $>10$ 。 of dorsiflexion with the knee extended, which improves past neutral with knee flexion (8). The angles of plantarflexion and dorsiflexion are indicated by positive and negative values, respectively.

Table 1

Patient information of the two Groups

\begin{tabular}{|lll|}
\hline & Modified group & Conventional group \\
\hline Patients & $34(42$ feet $)$ & 38 (42 feet) \\
\hline Male/Female & $2 / 32$ & $2 / 36$ \\
\hline Hallux valgus & $26(34$ feet $)$ & 30 (34 feet) \\
\hline Flatfoot & $7(7$ feet $)$ & 6 (6 feet) \\
\hline Replacement of metatarsophalangeal joint & $1(1$ feet $)$ & 2 (2 feet) \\
\hline
\end{tabular}

\section{Conventional Strayer procedure}

The skin incision for the Strayer procedure is made at the level of the musculotendinous junction of the gastrocnemius in line with the posterior border of the medial malleolus. The level of the incision has been described as $2 \mathrm{~cm}$ distal to the gastrocnemius indentation.(16) The gastrocnemius and soleus fascial 
layers are bluntly dissected down and the gastrocnemius fascia is transected one to two fingerbreadths distal to the musculotendinous junction without disruption of the soleus.

\section{Procedures of modified comprehensive technique}

Preoperatively, at plantarflexion position, color Doppler ultrasound was utilized to detected the musculotendinous junction of the well-placed affected foot, and the musculotendinous junction was marked on the skin. The center of the modified incision should be located approximately $3 \mathrm{~cm}$ to the vertical line of the medial border of the tibia.

Intraoperatively, place the affected foot at extreme plantarflexion, a 3-cm vertical incision was made through skin at the previously marked modified incision. The subcutaneous tissue and superficial fascia were bluntly dissected down. The gastrocnemius musculotendinous junction was cut off along the borders of medial and lateral heads of gastrocnemius, respectively. Then the ankle was forcefully held in dorsiflexion prolonging the gastrocnemius musculotendinous junction to reach a satisfying dorsiflexion angle. Thus, the avulsion was located distally to the skin and soft tissue incision to effectively avoid direct contact of avulsion and incision to reduce adhesion formation. Then fat and subcutaneous tissue were sutured by absorbable thread conventionally.

Postoperatively, the affected leg was immobilized in a plaster while the ankle was kept at neutral dorsiflexion for 2-3 weeks. The patients were encouraged to perform Achilles tendon and gastrocnemiusstretching exercises and ankle motion exercises during intermittent removal of plaster to further prevent scarring and adhesion formation.

\section{The principle of modified comprehensive technique to prevent postoperative scar adhesion}

The color Doppler ultrasound was utilized to detected the musculotendinous junction and the musculotendinous junction was marked on the skin at extremely plantarflexion position (Fig. 1, location A), then the musculotendinous junction was marked again during extremely dorsiflexion (Fig. 2, location B).

According to the distance between $A$ and $B$, posterior medial edge of the tibia can be palpated, and the longitudinal or small arc incision was performed at the vertical line about $3 \mathrm{~cm}$ from the posterior medial edge. The incision can be extended regrading the intersection of incision and line $A$ as the center but avoiding any overlapping of incision and line $B$. With ankle plantarflexion, the gastrocnemius musculotendinous junction was exposed and cut off sharply. With extreme dorsiflexion, the lower edge of the gastrocnemius muscle slid to B (Fig. 2), and the musculotendinous junction was prolonged and extended to the distal end ( $C$ in Fig. 2). Thus, there was no direct contact between the incision $D$ and extension area $C$, effectively preventing postoperative scar adhesions. Noteworthily, line E (Fig. 1) is the position of sural nerve, and it should be protected during the surgery thus exposure was not recommended. 
The diagnostic criteria of scarring and adhesion formation: skin and soft tissue on operative incision scar moved with dorsiflexion and plantarflexion of the ankle joint, affecting the cosmetic appearance or patients complained about local stiffness and discomfort.

\section{Statistical analysis}

The Statistical Package for the Social Sciences (version 19.0; SPSS, Inc, an IBM Company, Chicago, IL) was used for the statistical analysis of data. The maximum passive dorsiflexion angles of the ankle joint preoperatively and postoperatively within the group and between the groups were expressed in mean \pm standard deviation $(\mathrm{x} \pm \mathrm{s})$ and analyzed by the paired $\mathrm{t}$ test. Postoperative complication (scarring and adhesion formation) between the groups was compared using $\chi^{2}$ test. A $P$ value of less than 0.05 was defined to be statistically significant.

\section{Results}

The mean follow-up was 13.6 months (12 to 18 months). All of them had obvious improvement in the maximum passive dorsiflexion angle of the ankle joint with knee extension. The mean ankle dorsiflexion significantly improved from $14.5^{\circ} \pm 2.5^{\circ}$ degrees preoperatively to $-18.7^{\circ} \pm 3.2^{\circ}$ degrees in comprehensive modified procedure group (Modified group) postoperatively $(P<0.05)$, while dorsiflexion improved from $15.2^{\circ} \pm 3.0^{\circ}$ to $-19.1^{\circ} \pm 3.9^{\circ}$ in conventional Strayer procedure group (Conventional group) $(P<0.05)$ (Table 2).

Table 2

Comparison of dorsiflexion angle of ankle joint with knee extension

\begin{tabular}{|lcccc|}
\hline & Modified group & Conventional group & T-Value & P-Value \\
\hline Preoperative angle & $14.5^{\circ} \pm 2.5^{\circ} \#$ & $15.2^{\circ} \pm 3.0^{\circ} *$ & 1.138 & 0.262 \\
\hline Postoperative angle & $-18.7^{\circ} \pm 3.2^{\circ} \#$ & $-19.1^{\circ} \pm 3.9^{\circ} *$ & 0.411 & 0.683 \\
\hline \#T-Value 63.93 P-Value $\mathbf{0 . 0 0 0 *}$ T-Value $\mathbf{4 5 . 8 8}$ P-Value $\mathbf{0 . 0 0 0}$ & & \\
\hline
\end{tabular}

One foot of $42(2.4 \%)$ in modified procedure group developed scar adhesion while 12 of $42(28.6 \%)$ in conventional Strayer procedure group, which showed a statistically significant difference in postoperative complication (scar adhesion) rate between two groups $(P<0.05)$. (Table 3$)$ 
Table 3

Comparison of postoperative local scarring and adhesion formation in both group

\begin{tabular}{|lllll|}
\hline & $\begin{array}{l}\text { Scar adhesion } \\
\text { (foot) }\end{array}$ & $\begin{array}{l}\text { Non-adhesion } \\
\text { (foot) }\end{array}$ & $\begin{array}{l}\text { Total } \\
\text { (foot) }\end{array}$ & $\begin{array}{l}\text { Adhesion } \\
\text { rate(\%) }\end{array}$ \\
\hline Modified group (foot) & 1 & 41 & 42 & 2.4 \\
\hline $\begin{array}{l}\text { Conventional group } \\
\text { (foot) }\end{array}$ & 12 & 30 & 42 & 28.6 \\
\hline Total (foot) & 13 & 71 & 84 & 15.5 \\
\hline P-Value 0.003 & & & & \\
\hline
\end{tabular}

\section{Discussion}

Currently, the commonly used procedure for releasing gastrocnemius aponeurosis was that the longitudinal incision on posterolateral lower leg was performed in the midpoint of the fibular head to the tip of the lateral malleolus (7-16). One of the common postoperative complications was local scar adhesion, in which skin and soft tissue of the incision scar moved during dorsiflexion and plantarflexion of the ankle joint, affecting the appearance and some patients complained about local stiffness and discomfort (16).

The possible mechanisms of the adhesion formations are as follows: the direct contact of incision tissue and the avulsion of the releasing gastrocnemius aponeurosis; the immobilization for a long period of time with ankle joint in neutral position by plaster postoperatively; the lack of related active and passive exercise of ankle joint for functional rehabilitation.(17) The first point was considered to be the main cause for it resulted in local scar hyperplasia and adhesion in the healing process. Therefore, we designed a modified incision monitored by color Doppler ultrasonography, avoiding the superficial incision of skin soft tissue overlapped with the deep releasing avulsion of gastrocnemius aponeurosis. Utilizing the modified technique, preferable results were achieved except for limited local scar adhesion rate of $2.4 \%$, possibly due to the overlap of the longer and lower incision with the avulsion of gastrocnemius release. Meanwhile one patient showed the symptom of gastrocnemius injury in both experimental and control group, which possibly due to the limited incision and excessive traction, and the patients recovered from the deficit within one month with neurotrophic medication. Generally, the ankle dorsiflexion angles (with knee extension) were restored to $-18.7^{\circ} \pm 3.2^{\circ}$ from the preoperative $14.5^{\circ} \pm 2.5^{\circ}$ $(P<0.01)$, which was consistent with the previous literature ${ }^{20}$ on the lengthening of gastrocnemius aponeurosis and improvement of ankle dorsiflexion angle. Thus, the present technique was proved to be reliable and effective. It is noteworthy that the novel modified comprehensive technique can effectively prevent the postoperative scar adhesion as well as lengthen the gastrocnemius muscle.

Intraoperative patient posture 
All the patients in our present study were suffering from foot pathology with various etiologies, and most of them were positioned supinely during the operation. Whereas the prone position was recommended for a clear exposure and convenient operation for surgical gastrocnemius recession. And the change of the position during the operation resulted in additional steps and prolonged intraoperative time. To the best of our knowledge, supine position with the operating table inclining to the affected side for about 30 degrees is better for operation. Meanwhile, extreme ankle plantarflexion and slight knee flexion is recommended to be maintained by assistant for better surgical exposure.

Incision technique

According to the mark of the musculotendinous junction by ultrasonography preoperatively, the intraoperative incision was made as proximal as possible, and then was extended to distal junction area appropriately after the blunt exposure of gastrocnemius layer by layer to ensure that the distal end of the superficial skin incision was slightly proximal to the junction area (as indicated by the arrow in Fig. 3). If the incision was extended too far, there will increase the risk of the contact of the skin incision and the release area of gastrocnemius aponeurosis. In general, $3 \mathrm{~cm}$ of vertical straight incision was enough; while for patients with high $\mathrm{BMI}$, the incision could be extended to the proximal end, or a small arc incision can be made with vertex orientating the lateral side. After completely transection of gastrocnemius musculotendinous junction, the ankle joint was placed in extremely dorsiflexion to mobilize the avulsion of the junction. The superficial surface of the avulsion and the distance between the proximal end of loose part and the skin incision can be seen through the surgical field when the recession was completed (as indicated by the arrow in Fig. 4). Intraoperatively, the gastrocnemius fascia was intact within the incision and the muscle was not exposed. Thus, limited postoperative adhesion can be formed with the undamaged fascia and fat.

\section{Conclusions}

our present study introduced a novel modified comprehensive technique for releasing non-convulsive contracture of gastrocnemius muscle aponeurosis and the comparisons of surgical efficiency and complications were made between conventional Strayer procedure and modified technique, demonstrating the advantages of modified technique, especially for avoiding local scar adhesion. This present technique is worthy of spreading in foot and ankle surgery.

\section{Declarations}

\section{Ethics approval and consent to participate}

This study was conducted with approval from the Ethics Committee of Dalian Second People's Hospital and was performed in accordance with the Declaration of Helsinki. Written informed consent was obtained from all participants. For participants below 18 years, informed consents were obtained from legal guardian. 


\section{Consent for publication}

We have obtained consent to publish from the participants.

\section{Availability of data and materials}

The patients' data were collected in the Dalian Second People's Hospital. The datasets analyzed during the current study are available from the corresponding author on reasonable request.

\section{Competing interests}

The authors declare that they have no competing interests

\section{Funding}

No funding was received in support of this work.

\section{Authors' contributions}

All authors have read and approved the final manuscript. JZ was involved in the study design, data collection, drafting and revising of the manuscript. JZ, XS and MG were involved in the data collection. JZ and XS were involved in the analysis and interpretation of data. MG was involved in the study design, data collection, analysis and interpretation of the data. ZQ was involved in the study design, drafting and revising of the manuscript and has given final approval.

\section{Acknowledgements}

Not applicable.

\section{References}

1. Cicchinelli LD, Pascual Huerta J, Garcia Carmona FJ, et al. Analysis of astrocnemius recession and medial column procedures as adjuncts in arthroereisis for the correction of pediatric pesplanovalgus:a radiographic retrospective study. Foot Ankle Surg 47(5):385-391, 2008.

2. Chan JY, Williams BR, Nair P, et al. The contribution of medializing calcaneal osteotomy on hindfoot alignment in the reconstruction of the stage II adult acquired flatfoot deformity. Foot Ankle Int 34(2):159-166, 2013.

3. Meszaros A, Caudell G. The surgical management of equinus in the adult acquired flatfoot. Clin Podiatr Med Surg 24(4):667-685, 2007.

4. DiGiovanni BF, Moore AM, Zlotnicki JP, et al. Preferred management of recalcitrant plantar fasciitis among orthopaedic foot and ankle surgeons. Foot Ankle Int 33(6): 507-512, 2012.

5. Kiewiet NJ, Holthusen SM, Bohay DR, et al. Gastrocnemius recession for chronic noninsertional Achilles tendinopathy. Foot Ankle Int 34(4): 481-485, 2013. 
6. Jastifer JR, Marston J. Gastrocnemius contracture in patients with and without foot pathology. Foot Ankle Int 37(11):1165-1170, 2016.

7. Gurdezi S, Kohls-Gatzoulis J, Solan MC. Results of proximal medial gastrocnemius release for Achilles tendinopathy. Foot Ankle Int 34(10):1364-1369, 2013.

8. Maskill JD, Bohay DR, Anderson JG. Gastrocnemius recession to treat isolated foot pain. Foot Ankle Int 31(1):19-23, 2010.

9. Strayer LM. Recession of the gastrocnemius: an operation to relieve spastic contracture of the calf muscles. J Bone Joint Surg Am 32A(3):671-676, 1950.

10. Pinney SJ, Hansen ST, Sangeorzan BJ. The effect on ankle dorsiflexion of gastrocnemius recession. Foot Ankle Int 23(1):26-29, 2002.

11. Dayer $\mathrm{R}$, Assal $\mathrm{M}$, Chronic diabetic ulcers under the first metatarsal head treated by staged tendon balancing: a prospective cohort study. J Bone Joint Surg Br 91(4): 487-493, 2009.

12. Vuillermin C, Rodda J, Rutz E, et al. Severe crouch gait in spastic diplegia can be prevented: a population-based study. J Bone Joint Surg Br 93(12):1670-1675, 2011.

13. Chen L, Greisberg J. Achilles lengthening procedures. Foot Ankle Clin 14(4):627-637, 2009.

14. Herzenberg JE, Lamm BM, Corwin C, Sekel J. Isolated recession of the gastrocnemius muscle: the Baumann procedure. Foot Ankle Int 28(11):1154-1159, 2007.

15. Laborde JM, Philbin TM, Chandler PJ, Daigre J. Preliminary results of primary gastrocnemius- soleus recession for midfoot charcot arthropathy. Foot Ankle Spec 9(2):140-144, 2016.

16. Pinney SJ, Sangeorzan BJ. Surgical anatomy of the gastrocnemius recession (Strayer procedure). Foot Ankle Int 25(4):247-250, 2004.

17. Barske HL, DiGiovanni BF, Douglass M, Nawoczenski DA. Current concepts review: isolated gastrocnemius contracture and gastrocnemius recession. Foot Ankle Int 33(10):915-921, 2012.

18. Meszaros A, Caudell G. The surgical management of equinus in the adult acquired flatfoot. Clin Podiatr Med Surg 24(4):667-685, 2007.

19. Molund M, Paulsrud O, Ellingsen Husebye E. Results after gastrocnemius recession in 73 patients. Foot Ankle Surg20(4):272-275, 2014.

20. Benny A, Balg F, Svotelis A. Reconstruction of overlengthening after gastrocnemius recession with an achilles tendon allograft: case report. Foot Ankle Int 37(11):1249-1254, 2016.

21. Roukis TS, Schweinberger MH, Monica H. Complications associated with uni-portal endoscopic gastrocnemius recession in a diabetic patient population: an observational case series. Foot Ankle Surg 49(1):68-70, 2010.

22. Sammarco GJ, Bagwe MR, Sammarco VJ, Magur EG. The effects of unilateral gastrocsoleus recession. Foot Ankle Int 27(7):508-511, 2006.

23. Nawoczenski DA, Barske $H$, Tome J, et al. Isolated gastrocnemius recession for achilles tendinopathy: strength and functional outcomes. J Bone Joint Surg Am 97(2):99-105,2015. 
24. DiGiovanni CW, Kuo R, Tejwani N, et al. Isolated gastrocnemius tightness. J Bone Joint Surg Am 84(6):962-970,2002.

25. Morales-Muñoz P, De Los Santos Real R, Barrio Sanz P, et al. Proximal gastrocnemius release in the treatment of mechanical metatarsalgia. Foot Ankle Int 37(7):782-789, 2016.

26. Saxena A, Widtfeldt A. Endoscopic gastrocnemius recession: preliminary report on 18 cases. J Foot Ankle Surg 43(5):302-306, 2004.

\section{Figures}

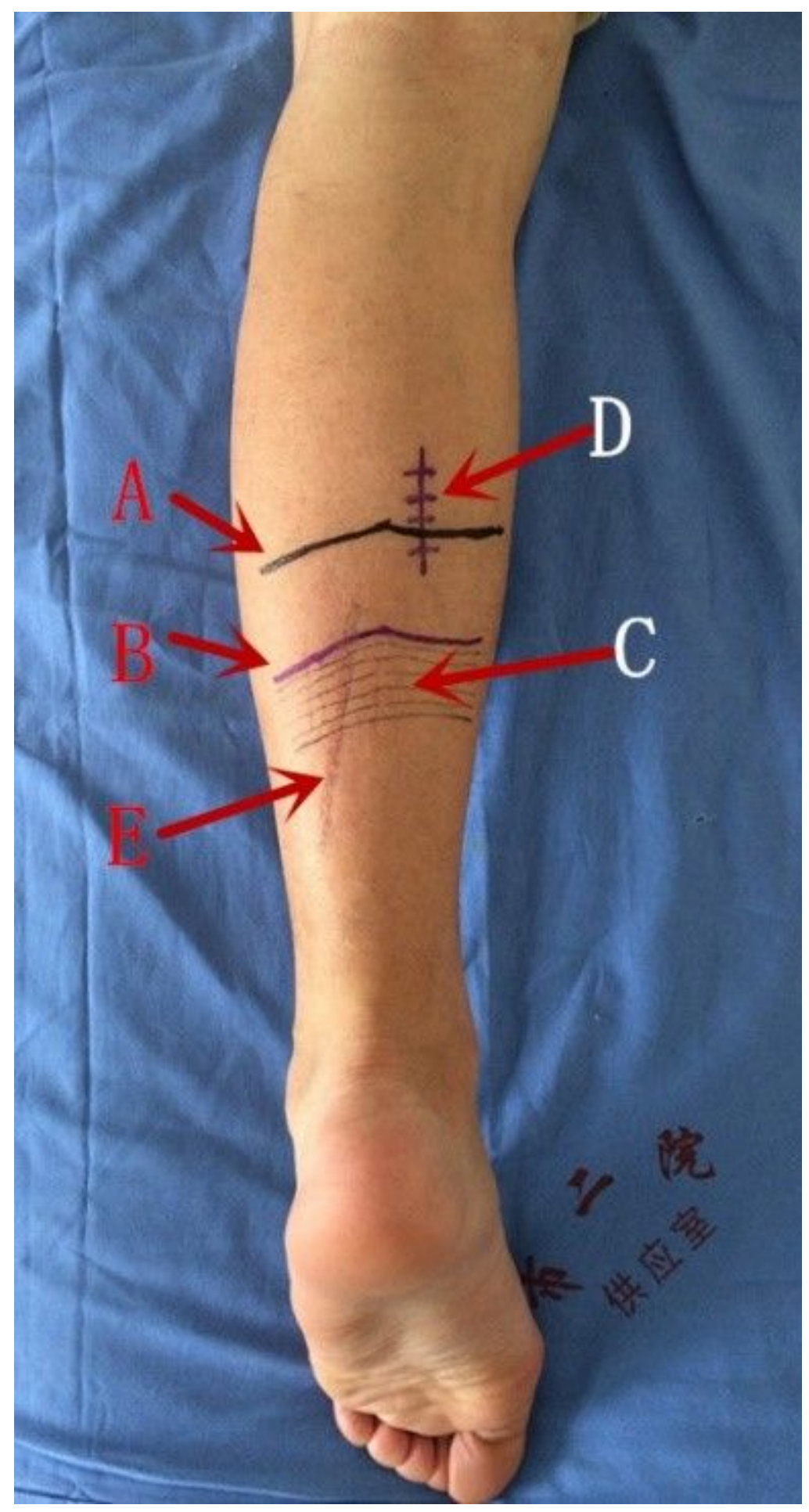




\section{Figure 1}

Preoperatively, color doppler ultrasound was utilized to detected the musculotendinous junction, and morphological and anatomical structures were marked on the skin: (A) The mark of gastrocnemius musculotendinous junction when ankle joint in extremely plantarflexion; (B) the mark of gastrocnemius musculotendinous junction when ankle joint in extremely dorsiflexion; (C) the mark of the junction avulsion area; (D) the mark of the surgical incision; (E) the mark of the sural nerve.

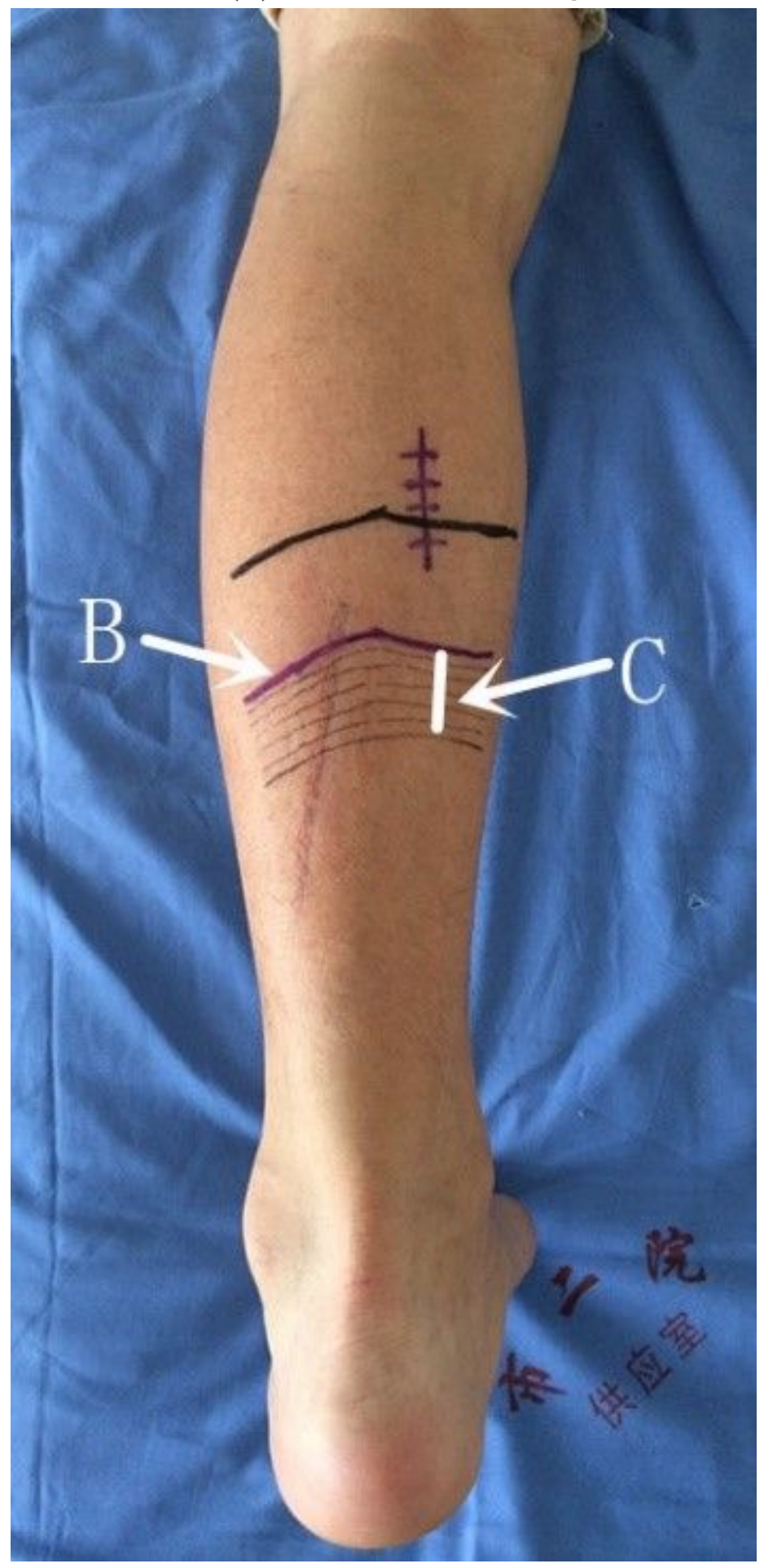

Figure 2 
When ankle joint in extremely dorsiflexion: (B) the mark of gastrocnemius musculotendinous junction; (C) the white line showed the fallen deep avulsion of the gastrocnemius musculotendinous junction.

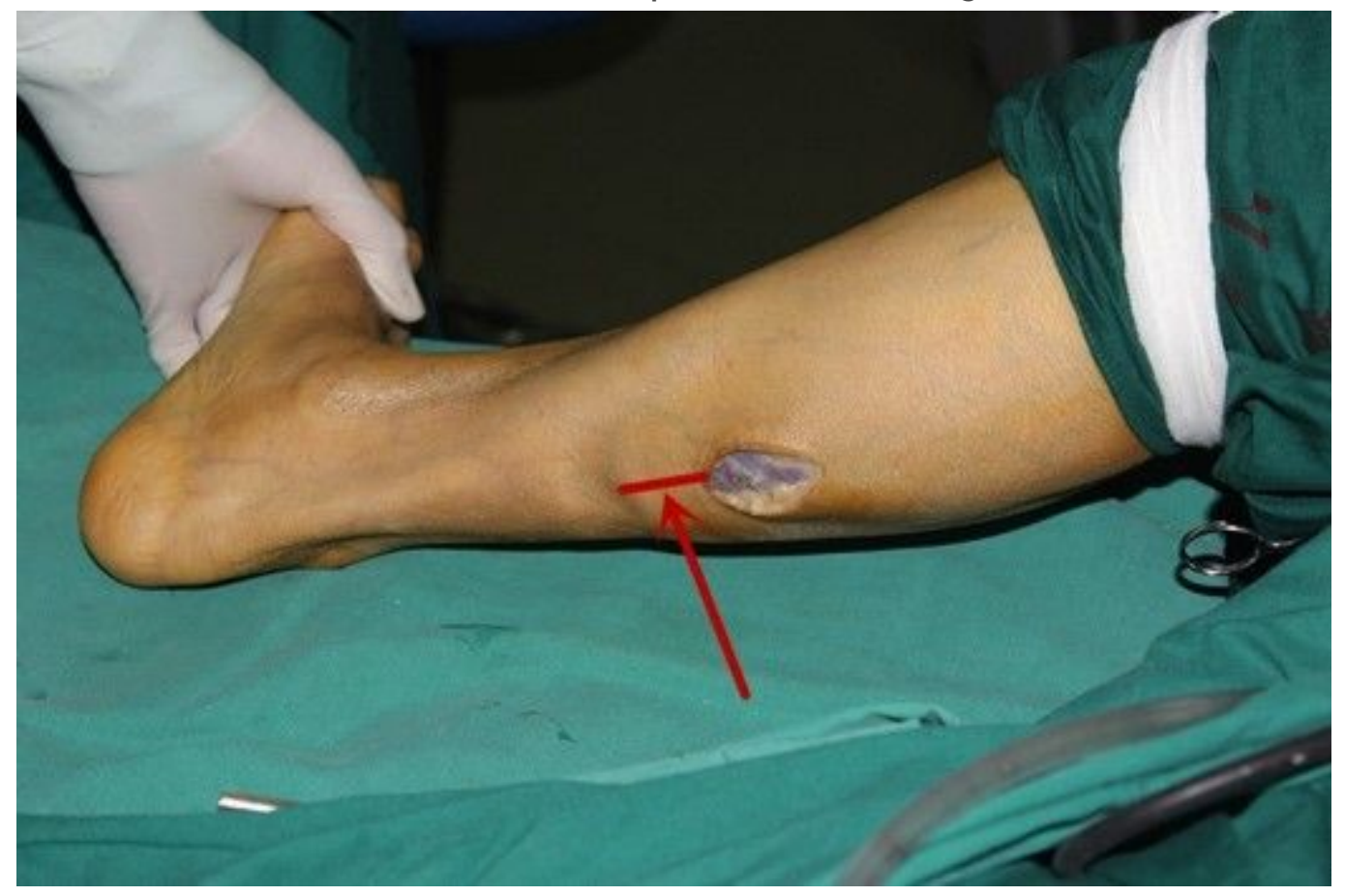

\section{Figure 3}

Intraoperative image of the Gastrocnemius Recession. The distance between distal end of the skin incision and the gastrocnemius musculotendinous junction. The intraoperative incision was made as proximal as possible, and then was extended to distal junction area appropriately after the blunt exposure of gastrocnemius layer by layer to ensure that the distal end of the superficial skin incision was slightly proximal to the junction area (red line).

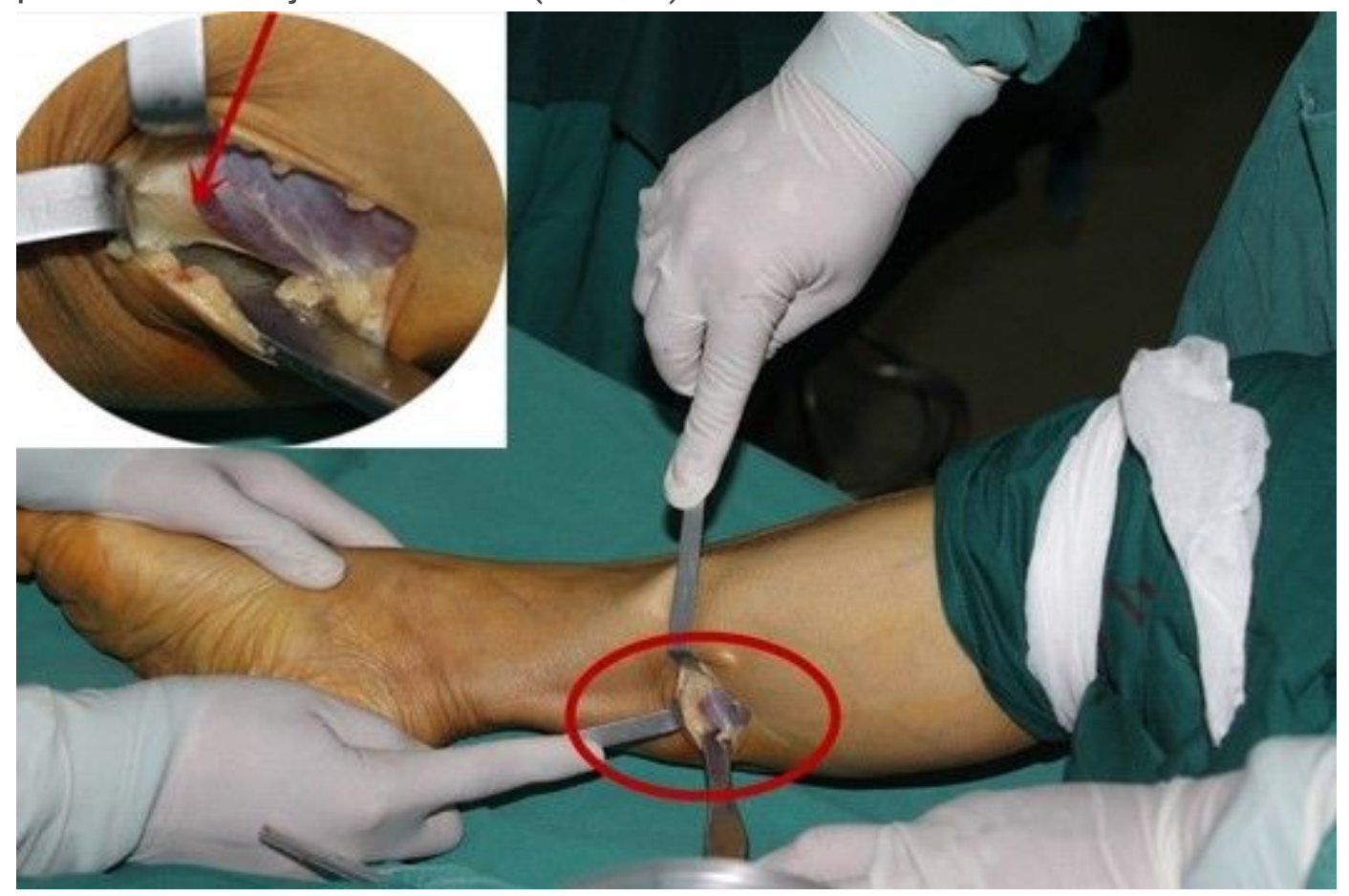




\section{Figure 4}

In plantarflexion, the incision of skin soft tissue and loose part. The enlarged image showed the gastrocnemius musculotendinous junction (red arrow). The superficial surface of the avulsion and the distance between the proximal end of loose part and the skin incision can be seen through the surgical field when the recession was completed. 